\title{
A Queueing Model for Node-disjoint Multipath Routing in Cluster-based Hierarchical Multimedia Sensor Networks
}

\author{
Qian Ye ${ }^{1, a}$, Yufei Wang ${ }^{2, \mathrm{~b}}$ and Zhengya Shan ${ }^{1}$ \\ ${ }^{1}$ College of Control Technology, Wuxi Institute of Technology, No.1600 Gaolangxi \\ Road, Wuxi, 214121, China \\ ${ }^{2}$ Jiangsu Electronic Information Products Quality Supervision Inspection \\ Research Institute, No.100 Jin Shui Road, Wuxi, 214073, China \\ ayeqian_80@163.com, ${ }^{b} w y f \_1999 @ 163 . c o m$
}

\begin{abstract}
Node-disjoint multiple routing is an effective mechanism for delivering rich multimedia in wireless multimedia sensor networks. And congestion is one of main problem must be addressed in routing scheme. Considering routing paths established are of noninterference and node-disjoint, each routing path can be viewed as a node. And then a queueing network model is introduced to abstract multiple type multimedia packets delivery process through node-disjoint routing paths in cluster based wireless multimedia sensor networks. At cluster header, QoS-aware traffic schedule scheme is adopted to dispatch packets of different priorities. The scheme consists of receiving buffer queue, traffic packets dispatching service based on generalized Erlang distribution, and prioritybased sending buffer queue. Then we model the optimal decision control process of multipath routing in congestion state. Finally, we simulate the proposed queueing network model with trust-based load balancing scheme. And the results reveal that our proposed model is more suitable for node-disjoint multiple paths to deliver rich multimedia information, such as video and images.
\end{abstract}

Keywords: sensor networks, queueing network, QoS-aware traffic schedule, load balancing, congestion control

\section{Introduction}

Multipath routing is the common mechanism adopted for providing reliability in Wireless Sensor Networks (WSNs)[1]; and it is also considered an effective approach to transmit large multimedia data in Wireless Multimedia Sensor Networks (WMSNs)[2]. Multimedia information in WMSNs are usually of rich types and burst. Tight Quality of Service (QoS) requirements in terms of packet losses, delay and jitter is main challenges for WMSNs [2-4]. So congestion is one of the main problem must be well addressed for WMSNs applications, such as multimedia surveillance networks, target tracking, environmental monitoring, and home automation systems.

Research efforts in solving the congestion problem of wireless sensor networks have been surveyed in [5]. The congestion type can be primarily classified into two major categories: contention-based congestion and buffer-based congestion. And congestion control protocols also can be classified into different categories based on different congestion detection and rate adjustment techniques[6], such as queue-assisted protocols, priority-aware protocols, topology formation protocols and resource control protocols. Congestion control protocols developed for traditional WSNs usually cannot satisfy high QoS requirements of video or audio stream, because the protocols are not content aware [6]. A two-stage WMSNs Congestion Control Protocol (WCCP) was introduced in [6]. The protocol is multimedia content aware and cross-layer. WCCP deployed a Group of Picture (GOP) size prediction method to predict congestion. However, few literature 
focuses on the congestion problem of the multiple path routing, especially for nodedisjoint multiple paths.

This paper establishes an open queueing network model for node-disjoint and noninterference multipath routing, which considers different multimedia traffic with different priorities. Then the proposed QoS-aware multiple traffic schedule scheme is adopted at the cluster header consists of priority-based non-preemptive queue and multimedia traffic packets dispatching service based on generalized Erlang distribution. Also, there is trustbased loading balancing among node-disjoint multiple routing paths. And optimal decision for multipath routing in congestion state is discussed based on markov decision processes.

\section{Traffic Classes with Multiple Priorities and Disjoint Multipath Routing}

In WMSNs, support and differentiated service for several different classes of applications are needed to be provided[2]. In this paper, delay and reliability requirements are two factors which decide the priority of the packet. As a result, the main traffic classes that need to be supported in WMSNs are shown in Table 1.

Table 1. Traffic Classes with Multiple Priorities[7]

\begin{tabular}{|c|c|c|}
\hline Traffic classes & Priority & Identifier \\
\hline $\begin{array}{c}\text { Real-time, } \\
\text { Loss-intolerant }\end{array}$ & High & 0 \\
\hline $\begin{array}{c}\text { Real-time, } \\
\text { Loss-tolerant }\end{array}$ & middle & 1 \\
\hline $\begin{array}{c}\text { Delay-tolerant, } \\
\text { Loss-intolerant } \\
\text { Delay-tolerant, } \\
\text { Loss-tolerant }\end{array}$ & low & 2 \\
\hline
\end{tabular}

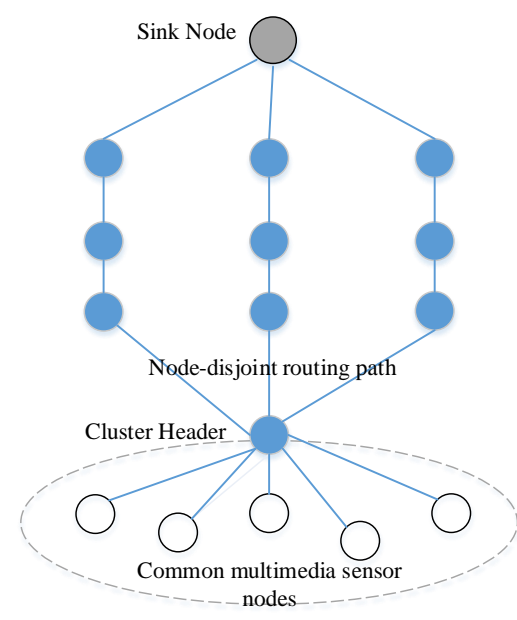

Figure 1. An Example of Disjoint Multipath Routing in Cluster Based WMSNs

As an effective routing scheme to provide high reliable packet delivery service, multipath mechanism we consider constructs a small number of alternate paths that are node-disjoint with each other. Disjoint multipath routing is used to establish multiple paths between each cluster-sink pair. Multimedia data generated by common multimedia sensor nodes are shipped to cluster head and then delivered to sink node through disjoint 
multiple paths which are assumed to be non-interfering. And non-interfering disjoint paths may be established if the number of paths is small. Figure 1 presents an example of disjoint multipath routing between one of cluster heads and sink node. As shown in Figure 1, three multiple paths do not share any common node nor any common link.

We do not take into account about how to establish node-disjoint multiple paths. And our works in this paper is over multiple paths that have been established.

\section{Queueing Network Model for Node-disjoint Routing}

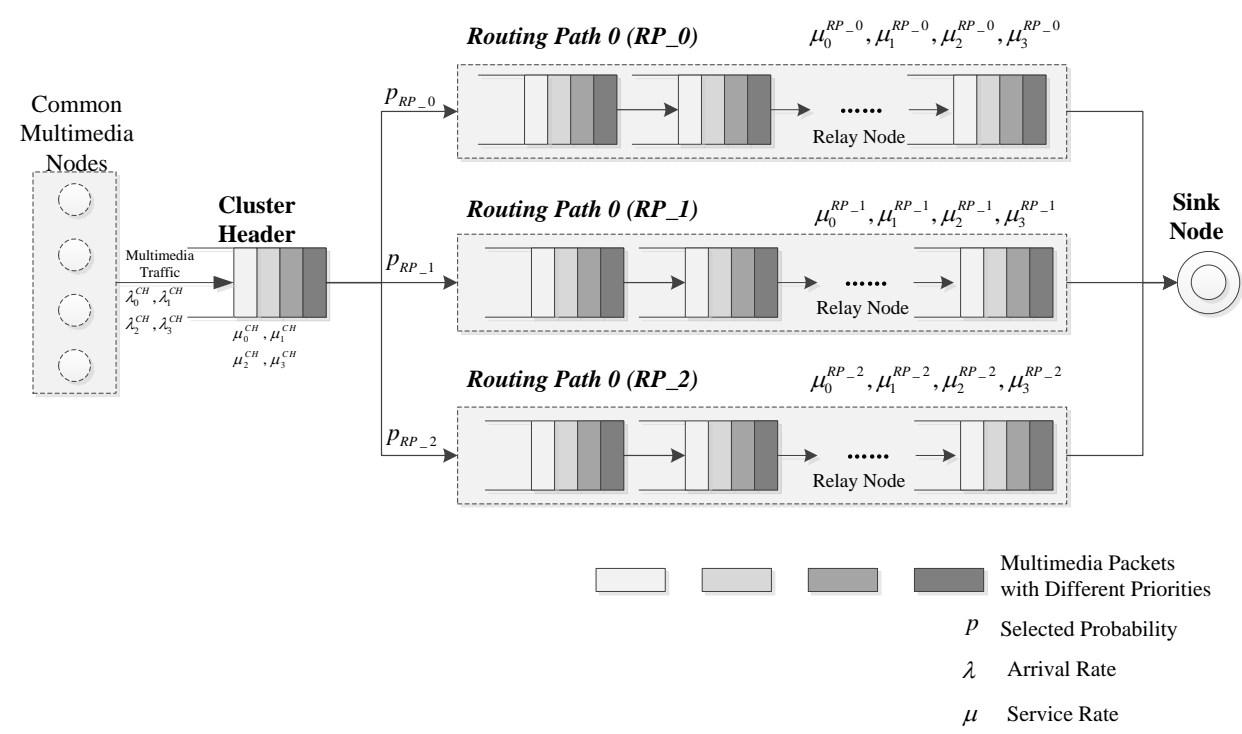

\section{Figure 2. Queueing Network Model for Node-disjoint Routing in Cluster based WMSNs}

The queueing network model for node-disjoint routing is given in Figure.2. The multiple routing paths are of non-interference and node-disjoint, so each node-disjoint routing path can be considered as a node. The open queueing network model of nodedisjoint routing system introduced in Figure.1 consists of four nodes which are Cluster Header, $R P \_0, R P \_1$, and $R P \_2$.

At Cluster Header, the arrival rates of packets of different traffic types shows in Table. 1 are $\lambda_{0}^{C H}, \lambda_{1}^{C H}, \lambda_{2}^{C H}$, and $\lambda_{3}^{C H}$; and the service rates are $\mu_{0}^{C H}, \mu_{1}^{C H}, \mu_{2}^{C H}$, and $\mu_{3}^{C H}$. Let us suppose that the service rates at the ithrouting path $R P P_{-} i$ are $\mu_{0}^{R P} P_{-}$, $\mu_{1}^{R P_{-} i}, \mu_{2}^{R P_{-} i}$, and $\mu_{3}^{R P_{-} i}$. If the probability to choose the $i$ th routing path $R P_{-} i$ to deliver the data packet leaving the Cluster Header is $p_{R P_{-} i}$, then the total packet arrival rate at routing path $R P_{-} i$ is $\left(\lambda_{0}^{C H}+\lambda_{1}^{C H}+\lambda_{2}^{C H}+\lambda_{3}^{C H}\right) p_{R P_{-} i}$. In which, $\sum_{i=0}^{n-1} p_{R P_{-} i}=1$.

The multimedia traffic packets may have forward routing paths. According to the queueing characteristic of the multiple routing paths, the node-disjoint routing system can be abstracted briefly as a feedforward queueing network. We make the following assumptions: (1) the nodes in sensor network have unlimited storage; (2) the nodes are service stations following the single exponential; (3) packets forward services among sensor nodes are mutual independence; $\lambda^{C H}=\lambda_{0}^{C H}+\lambda_{1}^{C H}+\lambda_{2}^{C H}+\lambda_{3}^{C H}$. And the traffic packet arrival rates at the four service stations in Figure.1, which are Cluster Header and three node-disjoint multiple 
routing paths, are $\lambda^{C H}, \lambda^{C H} p_{R P_{-} 0}, \lambda^{C H} p_{R P_{-} 1}$, and $\lambda^{C H} p_{R P_{-} 2}$. Let $Q_{C H}, Q_{R P_{-} 0}, Q_{R P_{-} 1}$, and $Q_{R P_{-} 2}$ respectively represent the numbers of traffic packets queueing at the four service stations with steady states. Then, the joint probability $P\left(k_{C H}, k_{R P_{-} 0}, k_{R P_{-} 1}, k_{R P_{-} 2}, t\right)$ is defined as:

$$
P\left(k_{C H}, k_{R P_{-} 0}, k_{R P_{-} 1}, k_{R P_{-} 2}, t\right)=P\left(Q_{C H}(t)=k_{C H}, Q_{R P_{-} 0}(t)=k_{R P_{-} 0}, Q_{R P_{-} 1}(t)=k_{R P_{-} 1}, Q_{R P_{-} 2}(t)=k_{R P_{-} 2}\right)
$$

The $M / M / 1$ queueing model can be used to abstract the marginal distribution of the packet numbers at Cluster Header. We ignore the different service rates for packets of different types temporarily. Let $\mu^{C H}, \mu^{R P_{-} 0}, \mu^{R P_{-} 1}$, and $\mu^{R P_{-} 2}$ respectively represent the average service rates at the four service stations. Then,

$$
P\left(Q_{C H}(t)=k_{C H}\right)=P_{C H}\left(k_{C H}\right)=\left(1-\rho_{C H}\right) \rho_{C H}^{k_{C H}} ; k_{C H}=0,1, \ldots \text {, where } \rho_{C H}=\lambda_{C H} / \mu^{C H} \text {. }
$$

On the basis of the Burke's theorem, for the $M / M / 1$ queueing model of packet arrival rate $\lambda^{C H}$, the process of packet leaving is the Poisson flow with parameter $\lambda^{C H}$ in steady state. Therefore, routing path $R P \_i$ can be considered as a service station following the $M / M / 1$ queueing model. Then, we have

$$
\begin{gathered}
P\left(Q_{R P_{-} \mathrm{i}}(t)=k_{R P_{-} i}\right)=P_{R P_{-} \mathrm{i}}\left(k_{R P_{-} \mathrm{i}}\right)=\left(1-\rho_{R P_{\mathrm{i}} \mathrm{i}}\right) \rho_{R P_{-} i}^{k_{R P_{\mathrm{i}}}} ; k_{R P_{-} \mathrm{i}}=0,1, \ldots, \text { where } \\
\rho_{R P_{-} i}=\lambda_{C H} * p_{R P_{-} \mathrm{i}} / \mu^{R P_{-} i}
\end{gathered}
$$

Based on the Burke's theorem, the packet departure process of a $M / M / 1$ queueing model and the content in queue are mutual independence. This means that packets at routing path $R P_{-} i$ and packets at Cluster Header are mutual independence. And then,

$$
P\left(k_{C H}, k_{R P_{-} \mathrm{i}}\right)=\left(1-\rho_{C H}\right)\left(1-\rho_{R P_{-} \mathrm{i}}\right) \rho_{C H}^{k_{C H}} \rho_{R P_{-}}^{k_{R P}} ; k_{C H}, k_{R P_{-} \mathrm{i}}=0,1,2, \ldots
$$

Considering the four traffic types show in Table.1, the proposed queueing system model for node-disjoint multiple routing consists of traffic schedule at Cluster Header, trust load balancing among multiple routing paths, and packet forwarding at relay sensor nodes.

First, multimedia traffic packets of different types produced by the common sensor nodes are delivered to Cluster Header. And the Cluster Headers cache the arrival packets in them receive buffer queues and shape the multimedia traffic through general Erlang distribution. Then, priority-based scheduler is used to dispatch the packets in receiving buffer queue to sending buffer queue with proper order.

Then, according to the state and trust level of each node-disjoint routing path, the transmission path of the packets in sending buffer queue is selected.

At each relay sensor node, priority-based queueing model is adopted to deliver multimedia packets of different types.

\section{QoS-aware Traffic Schedule Scheme in Cluster Header}

In this section, we will introduce the QoS-aware traffic schedule scheme at the Cluster Header as shown in Figure.3. The proposed traffic schedule model for cluster header consists of receiving buffer queue, traffic packets dispatching service based on generalized Erlang distribution, and priority-based sending buffer queue. 


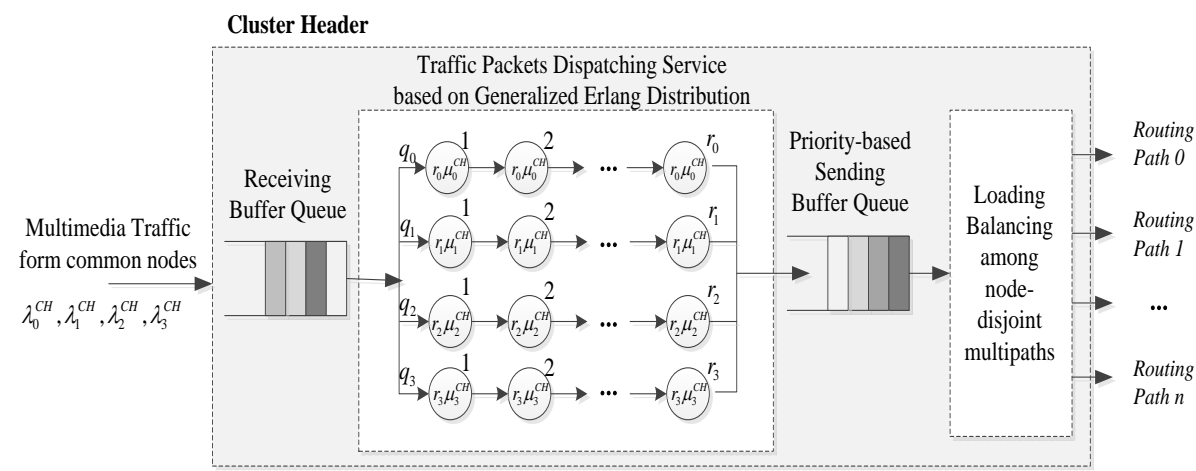

Figure 3. Traffic Schedule Model for Cluster Header

(1) Priority non-preemptive queue

The priority non-preemptive queue model is employed on the Cluster Header and each relay node in node-disjoint routing path. In the proposed traffic control scheme, priority scheduler is designed for dispatching packets of different traffic types based on their priorities. The incoming multimedia packets are cached in receiving buffer queue at Cluster Header and relay nodes. A classifier is adopted to check the packet type. In each transmission window[8], the priority scheduler determines the order of packets into sending buffer queue.

(2) Multimedia traffic scheduling based on general Erlang distribution

Different multimedia traffic encoded in application layer, such as video frames (I, $\mathrm{P}$ and $\mathrm{B}$ ), are encapsulated into large number of packets in transport layer. Every frame is split into packets of different numbers. Compared with $\mathrm{P}$ and $\mathrm{B}$ frames, I frame is usually split into more number of packets because of the encode characteristic. In multimedia sensor networks, traffic of different types have different priorities because they have different QoS requirements. The multimedia traffic shaping system based on general Erlang distribution is used to adjust the inter-departure time of packets of different types at the cluster header. This method can be used to avoid congestion. And this congestion control scheme makes use of the internal time correlation among the traffic packets of different types at the cluster header.

According to the four traffic classes given in Table.1, the general Erlang distribution has four parallel levels $(l=0,1,2,3)$, and the level $l$ consists of $r_{l}$ phases with exponentially distributed time and service rate $r_{l} \mu_{l}^{C H}$. Each level $l$ is selected with probability $q_{l}$, and $\sum_{l=0}^{3} q_{l}=1$. Then, the pdf (probability density function) [9] is:

$$
f_{X}(x)=\sum_{l=0}^{3} q_{l} * \frac{r_{l} \mu_{l}^{C H}\left(r_{l} \mu_{l}^{C H} x\right)^{r_{l}-1}}{\left(r_{l}-1\right) !} * e^{-r_{l} \mu_{l}^{C H} x}, x \geq 0
$$

The four parallel levels of the general Erlang distribution is corresponding to the four traffic classes in Table.1 with different QoS requirements (priorities). Packets of different classes have different service schedule time (inter-departure time) at the cluster header:

Level 0 of the general Erlang distribution is assigned to real-time and loss-intolerant packets. And the packets at this level will have the least inter-departure time.

Level 1 of the general Erlang distribution is assigned to real-time and loss-tolerant packets. And the packets at this level will have the less inter-departure time.

Level 2 of the general Erlang distribution is assigned to delay-tolerant and lossintolerant packets. And the packets at this level will have the larger inter-departure time. 
Level 3 of the general Erlang distribution is assigned to delay-tolerant and loss-tolerant packets. And the packets at this level will have the largest inter-departure time.

Let $X_{l}$ is the average inter-departure time of the packets in level $l$ and $\operatorname{Var}\left(X_{l}\right)$ is the variance, then:

$$
\begin{gathered}
\mu_{l}^{C H}=\frac{1}{\overline{X_{l}}} \\
r_{l}=\left\lceil\frac{\bar{X}_{l}^{2}}{\operatorname{Var}\left(X_{l}\right)}\right\rceil
\end{gathered}
$$

\section{Optimal Decision Control for Multipath Routing in Congestion State}

In this section, we focus on the optimal decision control scheme for multiple routing paths in congestion state. As shown in Figure.3, the model consists of optimal control and trust-based load balancing scheme.

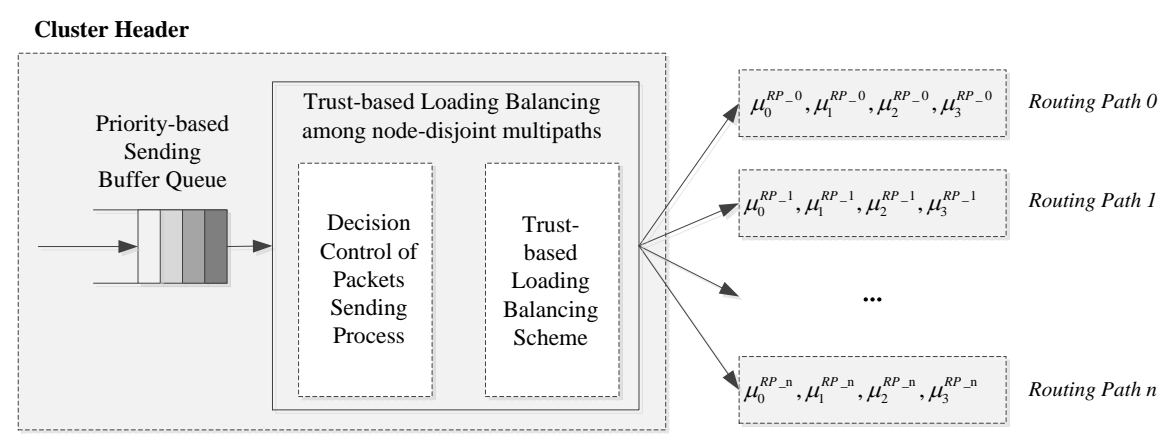

\section{Figure 4. Optimal Control and Trust-based Load Balancing Scheme for Multipath Routing}

Multiple node-disjoint routing paths can be considered as multiple service stations. A routing path is called occupied when the routing path is in congestion state. That is, packets have occupied the service station. To simply the discussion, we assume that the distribution of the congestion (service) time of each routing path follow the negative exponential distribution with parameter $\mu^{R P}$. The input of the distribution is $m(m=4)$ type's packets with different priorities, as shown in Figure.1. Let $F(t)$ is the distribution function which representatives the distribution of the packets arrival interval. When data packet arrivals, the probability of packet class falling into the $l(l=0,1,2,3)$ type is $e p_{l}$, and $e p_{l}>0, \quad \sum_{l=0}^{m-1} e p_{l}=1$.

The overall performance of the node-disjoint multiple routing paths in multimedia sensor networks can be evaluated through the QoS provided for the multimedia traffic coexist in the network, and the amount of data that can be transmitted. Then multiple routing path are abstracted as a set of benefit generated when successfully routing a multimedia packet, and the packet with different QoS requirement generates different benefit. Let $h_{l}$ represents the benefit generated through successfully routing packet of type $l(l=0,1,2,3)$. According to the packet priorities, we have $h_{0}>h_{1}>h_{2}>h_{3}>0$. 
For a routing path, congestion means the routing path (i.e. service station) is occupied. When all the routing paths in network are in congestion, it implies that all the service stations are occupied. And the arrival packets will be dropped. When packet arrivals, if any routing path is not in congestion, the model should decide whether to route the arrival packet or not. If the arrival packet is routed, the model will have the corresponding benefit. If not, the routing path may be hold for routing the arrival packet with higher priority.

In case the congestion is happened, the optimal decision control of the multiple routing paths with the same service rate is the problem to achieve the optimal control of the input process. That is, the model gets the maximum benefits form all the multiple routing paths in the multimedia sensor networks through controlling the decision of accept or reject the arrival packet. Suppose there are $n$ node-disjoint routing paths, the optimal input control of multiple routing in congestion can be converted to a markov decision processes [10].

We call node-disjoint routing path is in state $i$, when there are $i$ routing paths are in congestion, in which $0 \leq i \leq n$. Each decision in decision space $D$ is a subset of $L=\{0,1, \cdots, m-1\}$, and according the multimedia traffic classes shown in Table.1, $L=\{0,1,2,3\}$. If decision $d=\{0,1\}$, then the arrival packets of 0 or 1 traffic class will be routed, and arrival packets of 2 and 3 traffic classes will not be routed. Suppose that decision space $D_{i}$ is corresponding to the state $i$, then $D_{i}$ is the set including all the subsets of $L=\{0,1, \cdots, \mathrm{m}-1\}$. If $i<n$, which means that not all the routing path is in congestion, then $\phi \notin D_{i}$. If $i=n, D_{n}=\phi$. This implies that all the routing paths cannot route packets successfully.

Let policy set $F=\left\{f=\left[\begin{array}{c}f_{0} \\ f_{1} \\ \vdots \\ f_{n}\end{array}\right]: f_{i} \in D_{i}, 0 \leq i \leq n\right\}$.

If node-disjoint routing is in state $i$, the packet of traffic class $l$ will be delivered only if $l \in f_{i}$.

When the $x$ packet of traffic class $l$ arrivals, let multiple routing state is $X_{x}^{-}, x=1,2, \cdots$. Suppose that decision $f$ is used to the arriving packets, then we have:

$$
X_{x+1}^{-}=\left\{\begin{array}{c}
X_{x}^{-}+1-v_{x}, l \in f_{X_{x}^{-}} \\
X_{x}^{-}, l \notin f_{X_{x}^{-}}
\end{array}\right.
$$

In which, $v_{x}$ is the number of routing paths which leave the congestion state within the time interval $\tau_{x}$ between the $x$ and $x+1$ arrival packets. Then $\left\{X_{x}^{-}, x=1,2, \cdots\right\}$ is a homogeneous markov chain. According to the theorem 5.1.1 in reference[10], the one-step transition probability matrix

$$
P_{f}(1)=\left[\begin{array}{cccc}
p_{00}\left(f_{0}\right) & p_{01}\left(f_{0}\right) & \cdots & p_{0 n}\left(f_{0}\right) \\
p_{10}\left(f_{1}\right) & p_{11}\left(f_{1}\right) & \cdots & p_{1 n}\left(f_{1}\right) \\
\vdots & \vdots & \vdots & \vdots \\
p_{n 0}\left(f_{n}\right) & p_{n 0}\left(f_{n}\right) & \cdots & p_{n 0}\left(f_{n}\right)
\end{array}\right]
$$


Specially, for the multipath routing consists of three node-disjoint routing path, we have

$$
P_{f}(1)=\left[\begin{array}{llll}
p_{00}\left(f_{0}\right) & p_{01}\left(f_{0}\right) & p_{02}\left(f_{0}\right) & p_{03}\left(f_{0}\right) \\
p_{10}\left(f_{1}\right) & p_{11}\left(f_{1}\right) & p_{12}\left(f_{1}\right) & p_{13}\left(f_{1}\right) \\
p_{20}\left(f_{2}\right) & p_{21}\left(f_{2}\right) & p_{22}\left(f_{2}\right) & p_{23}\left(f_{2}\right) \\
p_{30}\left(f_{3}\right) & p_{31}\left(f_{3}\right) & p_{32}\left(f_{3}\right) & p_{33}\left(f_{3}\right)
\end{array}\right]
$$

where,

$$
p_{i j}\left(f_{i}\right)=\left\{\begin{array}{c}
\sum_{l \in f_{i}} e p_{l} \int_{0}^{\infty}\left(\begin{array}{c}
j+1 \\
j
\end{array}\right)\left(1-e p^{-\mu^{R P} t}\right)^{i+1-j} e p^{-j \mu^{R P} t} d F(t)+\sum_{l \in f_{i}} e p_{l} \int_{0}^{\infty}\left(\begin{array}{l}
i \\
j
\end{array}\right)\left(1-e p^{-\mu^{R P} t}\right)^{i-j} e p^{-j \mu^{R P t}} d F(t), \mathrm{j} \leq \mathrm{i}<\mathrm{n}, \\
\sum_{l \in f_{i}} e p_{l} \int_{0}^{\infty} e p^{-(i+1) \mu^{R P t}} d F(t), j=i+1, i<n, \\
0, j>i+1, i<n, \\
\int_{0}^{\infty}\left(\begin{array}{c}
n \\
j
\end{array}\right)\left(1-e p^{-\mu^{R P} t}\right)^{n-j} e p^{-j \mu^{R P} t} d F(t), j \leq n .
\end{array}\right.
$$

For each decision $d \in D_{i}$, the benefit function $h(i, d)$ is defined as:

$$
h(i, d)=h(d)=\sum_{l \in d} e p_{l} h_{l}, l=0,1,2,3
$$

Suppose that the initial state of the multipath routing $X_{1}^{-}=i$ and decision is $f$ when the first packet arriving. Let $H_{x}(i, f)$ be the total benefits gained after the $x$ th packet arrivals. Then the long-term average total expected benefit value $E(i, f)$ of multipath routing in congestion state can be calculated by

$$
E(i, f)=\lim _{X \rightarrow \infty} \frac{1}{X} \sum_{x=1}^{X} \sum_{j=0}^{n} h\left(f_{j}\right) \cdot P\left\{X_{x}^{-}=\left.j\right|_{X_{1}^{-}=i ; f}\right\}
$$

In which, $P\left\{X_{x}^{-}=\left.j\right|_{X_{1}^{-}=i ; f}\right\}$ is element on line $i$ column $j$ of matrix $\left[P_{f}(1)\right]^{x-1}$ because $\left\{X_{x}^{-}, x=1,2, \cdots\right\}$ is homogeneous markov chain.

$$
\text { Let } E(f)=\left[\begin{array}{c}
E(0, f) \\
E(1, f) \\
\vdots \\
E(n, f)
\end{array}\right], \quad h(f)=\left[\begin{array}{c}
h\left(f_{0}\right) \\
h\left(f_{1}\right) \\
\vdots \\
h\left(f_{n}\right)
\end{array}\right] .
$$

Then $E(i, f)$ can be expressed as matrix form:

$$
E(f)=\lim _{X \rightarrow \infty} \frac{1}{X} \sum_{x=1}^{X}\left[P_{f}(1)\right]^{x-1} h(f)
$$

Any two states of homogeneous markov chain $\left\{X_{x}^{-}, x=1,2, \cdots\right\}$ are interoperable, and elements on the diagonal line of $P_{f}(1)$ is not equal to zero. So $\left\{X_{x}^{-}, x=1,2, \cdots\right\}$ is irreducible and aperiodic. The congestion states of the routing paths is limited 
because of the routing paths is numbered. And then $E(f)$ exits. Let $\lim _{X \rightarrow \infty}\left[P_{f}(1)\right]^{x}=P_{f}^{\prime \prime}>0$, then we have $E(f)=P_{f}^{\prime \prime} \cdot h(f)$.

In multimedia sensor networks with multiple traffic classes, the optimal control problem of node-disjoint routing paths in congestion can be described as finding the optimal policy $f$ to maximize all the coordinates of $E(f)$ which is the expectation of the long-term average total benefits gained by delivering multimedia packets with different priorities through multiple routing paths. Reference[10] provides an algorithm to find optimal policy in the markov decision process. And the algorithm can be used to solve $f$.

According to the optimal policy $f$ and the arrival multimedia packet type, the cluster head decides whether to route the arrival packet. If the arrival packet type $l \in f$ and $n-i \geq 2$ which means that more than two node-disjoint routing paths are not in congestion and can route the arrival packet, then one of the routing paths will be selected to transmit the arrival packet.

Trust-based load balancing scheme [11] is also adopted for QoS-guaranteed multimedia transmission through multiple node-disjoint routing paths in WMSNs.

\section{Simulation}

We adjust the inter-departure time of the different type packets at the cluster head based on the general Erlang distribution. Figure.5 shows the value of the total probability density function of the traffic service time considering different collect probabilities. And the parameters are

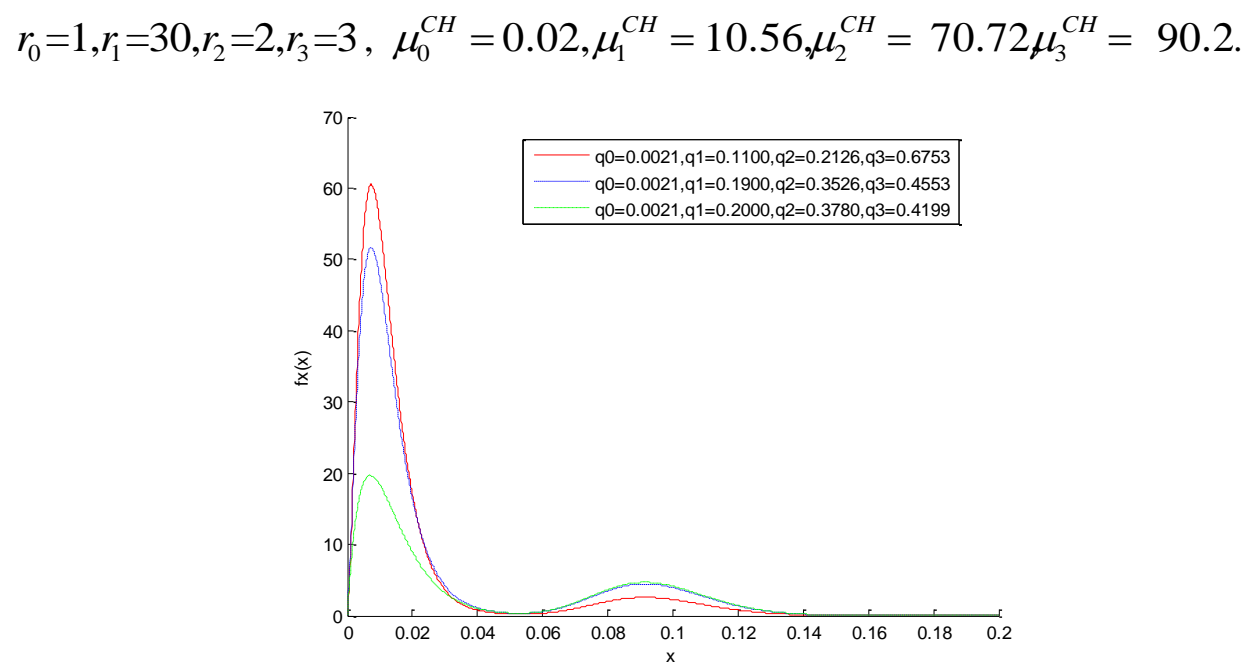

\section{Figure 5. Value of the Total Probability Density Function of the Traffic Service Time}

We simulate the load balancing scheme based on the proposed queueing model in NS2, and the network topology was created as Figure 1. The classic foreman_qcif.yuv, provided by TNK research institute, was selected for test sequence, the format of which was $176^{*} 144$ pixels. The test sequence was composed of 400 frames. The original frames were encoded to MPEG-4. And the format of the GOP (Group of Pictures) is IPBBPBBPIP.

Based on network topology shown in Figure 1, we analyze and compare the performance of the proposed load balancing scheme with WCCP[6] and 
XLP[12].The simulation results as shown in Figure 6-8 are average loss number of I-packets, P-packets, and B-packets.

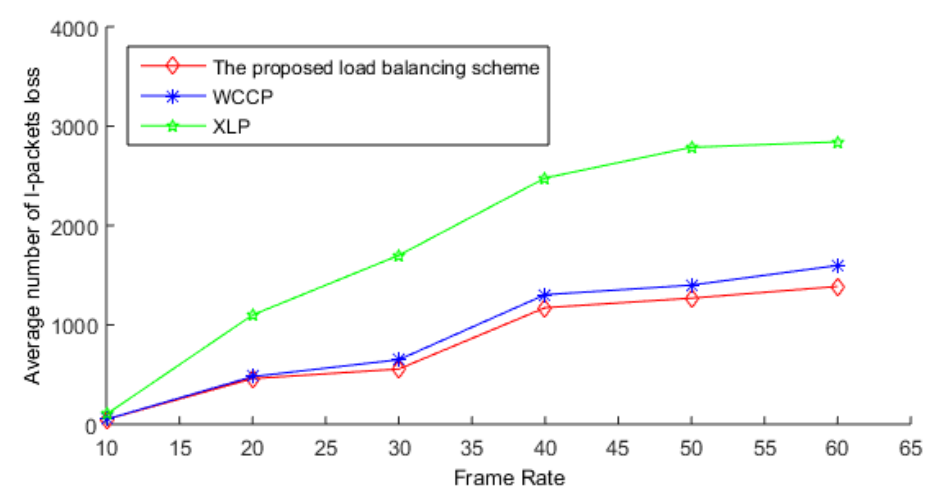

Figure 6. Average Number of I-packets Loss

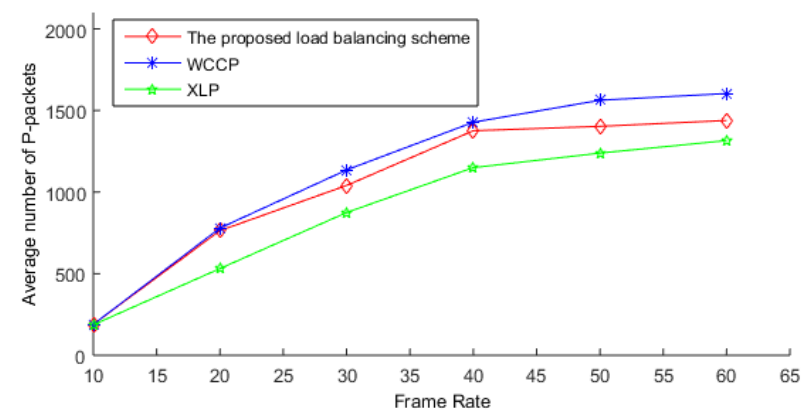

Figure 7. Average Number of P-packets Loss

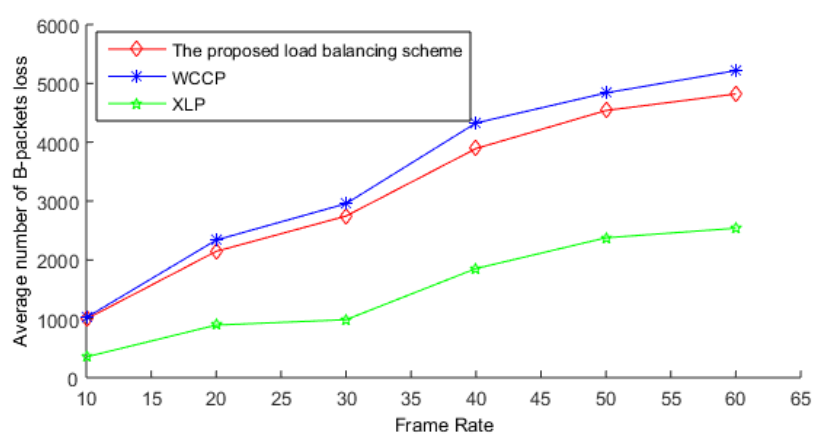

Figure 8. Average Number of B-packets Loss

As can be seen from the Figure 6-8, comparing with WCCP[6] and XLP[12], the I-packets delivery rate of proposed load balancing method is higher than that of XLP. And the P-packets and B-packets delivery rates of proposed load balancing method are lower than those of XLP. Because the proposed load balancing method and WCCP first deliver I-packets with higher priorities, and then the P-packets and B-packets. The decoded image quality is usually depended on the transmission quality of the I-frames.

On the other hand, in our proposed load balancing scheme, I-packets, P-packets and B-packets will be delivered through different routing paths according to their different priorities. And I-packets will be delivered through the routing path with most trusted level and highest QoS. Therefore, the I-packet, P-packet, and B-packet 
correct delivery rates of the proposed load balancing scheme is better than those of WCCP.

\section{Acknowledgement}

This study is supported by the Research Program of Wuxi Institute of Technology (Research on Key Technologies of information security for Industrial Control Systems).

\section{References}

[1] S. Misra, M. Reisslein, and G. Xue, "A survey of multimedia streaming in wireless sensor networks", IEEE Communications Surveys \& Tutorials, vol. 10, (2008), pp. 18-39.

[2] I. F. Akyildiz, T. Melodia and K. R. Chowdhury, "A survey on wireless multimedia sensor networks", Computer Networks, vol. 51, pp. 921-960, (2007).

[3] O. B. Akan, P. Frossard, Q. Zhang, and N. Jayant, "Special issue on wireless multimedia sensor networks", Computer Networks, vol. 52, pp. 2529-2531, (2008).

[4] L. Atzori, T. Dagiuklas, and C. Politis, "Special issue on multimedia over ad-hoc and sensor networks", Mobile Networks and Applications, vol. 13, pp. 243-245, (2008).

[5] M. Kafi, D. Djenouri, J. Ben-Othman and N. Badache, "Congestion Control Protocols in Wireless Sensor Networks: A Survey", Communications Surveys \& Tutorials, IEEE, vol. PP, (2014), pp. 1-22.

[6] S. M. Aghdam, M. Khansari, H. R. Rabiee and M. Salehi, "WCCP: A congestion control protocol for wireless multimedia communication in sensor networks", Ad Hoc Networks, vol. 13, Part B, (2014), pp. 516-534, 2//.

[7] Y. Qian, W. Meng and W. Yufei, "Traffic Scheduling Scheme for Disjoint Multipath Routing Based Wireless Multimedia Sensor Networks", in Services Computing Conference (APSCC), 2010 IEEE AsiaPacific, (2010), pp. 388-393.

[8] I. Politis, M. Tsagkaropoulos, T. Dagiuklas and S. Kotsopoulos, "Power efficient video multipath transmission over wireless multimedia sensor networks", Mob. Netw. Appl., vol. 13, (2008), pp. 274-284

[9] G. Bolch, S. Greiner, H. de Meer and K. S. Trivedi, "Queueing networks and Markov chains: modeling and performance evaluation with computer science applications", John Wiley \& Sons, (2006).

[10] T. YInghui and T. Xiaowo, "Queueing Theory-Foundation and Analyzing Technology", Beijing: Science Press, (2006).

[11] Y. Qian and W. Meng, "A QoS-aware trust model for multipath load balancing in multimedia sensor networks", Journal of Software Engineering, vol. 9, (2015), pp. 50-65.

[12] M. C. Vuran and I. F. Akyildiz, "XLP: A Cross-Layer Protocol for Efficient Communication in Wireless Sensor Networks", Mobile Computing, IEEE Transactions on, vol. 9, (2010), pp. 1578-1591. 
International Journal of Future Generation Communication and Networking Vol. 9, No. 12 (2016) 